Ann. Biol. anim. Bioch. Biophys., I970, 10 (4), 549-566.

\title{
ACTION D'IMPLANTS DE BENZOATE D'CESTRADIOL DANS L'HYPOTHALAMUS SUR LE COMPORTEMENT D'GESTRS DE LA BREBIS OVARIECTOMISÉE
}

\author{
J.-P. SIGNORET \\ avec la collaboration technique de Colette Lavenet et P. ORgeur \\ Station de Recherches sur la Physiologie de la Reproduction, \\ Centre de Recherches de Tours, 37 - Nouzilly \\ Institut national de la Recherche agronomique
}

\section{RÉSUMÉ}

Des implants de benzoate d'œstradiol portés par une tige d'acier inoxydable ont été mis en place dans l'hypothalamus de brebis ovariectomisées recevant ou non des injections intramusculaires de progestérone.

Malgré une action systémique mise en évidence au niveau du tractus génital (augmentation du poids de l'utérus, cornification du frottis vaginal), seuls les implants localisés à l'hypothalamus antérieur préoptique latéral font apparaître des réponses d'œstrus en l'absence de progestérone ou pendant les injections de cette hormone. Par contre, l'effet facilitant du traitement progestéronique fait apparaître chez tous les animaux ayant reçu un implant d'ostrogène une réponse sexuelle environ deux jours après la fin de ce traitement.

Des éléments de comportement sexuel pseudo-mâle sont apparus chez la plupart des brebis ayant reçu des implants hypothalamiques d'hormone. Les réponses de comportement sexuel femelle ou mâle sont exceptionnelles chez les divers témoins (implants de benzoate d'œestradiol dans le cortex, implants de cire dans l'hypothalamus).

A défaut d'un centre précis, il a été possible de mettre en évidence une zone privilégiée pour l'action des œstrogènes au niveau hypothalamique. L'apparition de réactions pseudo-mâles confirme chez la brebis où ces réactions sont spontanément exceptionnelles, la présence des deux schémas de comportement sexuel mâle et femelle et leur possibilité de mise en ceuvre simultanée par la même hormonc.

\section{INTRODUCTION}

L'apparition du comportement d'œstrus à la suite d'implants ou de microinjections d'œstrogènes dans l'hypothalamus a été mise en évidence chez la Chatte (Harris et MrchaEl, I958, I964; Michaei, I961, I962; Sawyer, I963), la Ratte (Lisk et WEIN, I962; LISK, I962, I965), la Lapine (PALKA et SAWYer, I964, I966). 
Or, chez la Brebis ovariectomisée, l'équilibre endocrinien qui déclenche 1'apparition de l'œstrus requiert la succession progestérone-œestrogène (RoBINson, 1954) alors que chez la Chatte les ostrogènes seuls sont suffisants (MICHAEL, et Sco'T'T, I957, I964) et que chez la Ratte un traitement de progestérone doit suivre les ostrogènes (Boling et BlandaU, I939; BEACH, I942). Le but de l'étude entreprise ici est d'envisager les possibilités d'une action locale des œstrogènes soit seuls, soit en relation avec un traitement systémique de progestérone.

\section{MATÉRIEL ET MÉTHODES}

Nous avons utilisé des brebis vierges de race Ile-de-France âgées de 16 à 20 mois et ovariectomisées au moins un mois avant le traitement expérimental.

\section{Préparation de l'implant}

La dureté relative de la pie-mère chez la brebis a nécessité l'utilisation d'aiguilles acérées pour éviter que celles-ci ne soient déviées lors de la descente dans le cerveau. De ce fait le cristal l'bormone a été déposé dans une gorge située au-dessus de la pointe de l'aiguille selon la figure I.

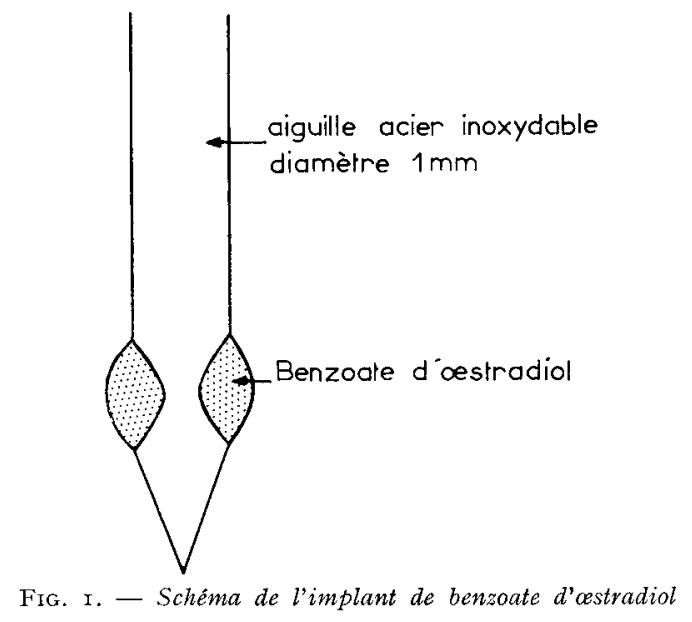

L'hormone - benzoate d'œstradiol - était fondue en chauffant avec précaution une petite quantité de poudre placée sur l'aiguille; dès la fusion le liquide occupait la place disponible dans la gorge. Après solidification, l'excès d'hormone débordant cette zone était gratté au scalpel sous la loupe binoculaire. La quantité totale d'hormone était déterminée par pesée de l'aiguille et a été trouvée égale à $3,27 \pm 0,18 \mathrm{mg}$.

Pour mettre en évidence une influence éventuelle de la lésion provoquée par la mise en place de l'aiguille, des implants témoins ont été réalisés en plaçant sur des aiguilles identiques une goutte de cire à trépanation d'un volume analogue à celui des implants d'œstrogènes.

\section{Mise en place de l'implant}

La brebis anesthésiée est placée dans un cadre stéréotaxique du type Horsley-Clarke adapté à l'espèce ovine. La carte des structures nerveuses correspondant aux coordonnées de l'appareil a été établie antérieurement au laboratoire pour des animaux de même âge, sexe et race. 
Pour atteindre des structures voisines du plan médian, il était nécessaire de réaliser des implants inclinés et non pas verticaux pour éviter de léser le sinus veineux médian.

Après que l'os aît été perforé à la fraise, la dure-mère est incisée et l'implant descendu au moyen d'une crémaillère au I/IO de $\mathrm{mm}$; la tige, maintenue en place avec du ciment dentaire, est ensuite coupée puis la peau est suturée.

Les implants ont toujours été effectués bilatéralement.

\section{Observations de comportement}

Le comportement sexuel a été mis en évidence par présentation quotidienne des femelles au bélier pendant $30 \mathrm{mn}$ ( 2 béliers par groupe de 30 brebis).

En outre, les réactions de comportement des femelles entre elles ont été observées une heure par jour au cours de 32 journées réparties sur la totalité de l'expérience. Un groupe de 43 brebis normales de même race et $d u$ même âge a été observé en même temps à titre de témoins. Les cycles sexuels de ces derniers animaux étant synchronisés par des progestagènes pour l'insémination artificielle, des observations ont eu lieu lorsque toutes les brebis étaient en œstrus ( 2 périodes de 2 jours) ou hors de l'cestrus ( 28 journées).

\section{Réponse vaginale}

Des frottis vaginaux ont été effectués régulièrement 5 jours de suite chaque semaine (MnokE et Robrnson, I957).

\section{Contrôle histologique}

Immédiatement après abattage, la tête des brebis a été perfusée au formol à ro p Ioo, le cerveau coupé après congélation à $50 \mu$ et coloré selon la technique de NissL. Une coupe est conservée tous les $0,5 \mathrm{~mm}$ pour repérer la localisation exacte de l'implant.

L'intégralité de l'ovariectomie a été vérifiée à l'abattage ; l'utérus a été pesé dans la deuxième expérience seulıment.

\section{Utilisation de l'hormone}

La quantité d'hormone restant après 7 mois d'implantation a été déterminée ${ }^{1}$ ) par la réaction de Kober avec extraction du chromogène rose par une solution de 2 p. Ioo de paranitrophénol dans le tétrachlorure d'acétylène suivant la technique d'ItTrich (I958-I960). Les lectures au spectrophotomètre ont été effectuées à trois longueurs d'ondes afin d'appliquer la correction d'AlEN : 5 Io, $54^{\circ}$ et $57^{\circ} \mathrm{m} \mu$, la densité optique corrigée (DOC) étant :

$$
\mathrm{DOC}=\mathrm{DO} 54^{\circ}-\frac{\mathrm{DO} 5 \mathrm{IO}^{\mathrm{O}}+\mathrm{DO} 57^{\circ}}{2}
$$

\section{PLAN EXPÉRIMENTAL}

Deux expériences successives ont été effectuées.

\section{Expérience $I$}

Cet essai préliminaire avait pour but de mettre en évidence l'effet d'implants localisés dans les différentes parties de l'hypothalamus comparé à celui d'implants témoins, soit dans le cortex cérébral, soit sous-cutané. L'effet local de la lésion induite par la mise en place de l'aiguille portant le benzoate d'œstradiol étant étudié par des implants de cire.

Trente-trois brebis ont reçu l'un des traitements suivants :

Traitements expérimentaux.

Implantation bilatérale de benzoate d'œstradiol dans l'hypothalamus antérieur ( 7 animaux) médian (9 animaux) ou postérieur ( 7 animaux).

(1) L'auteur remercie M. Fèvre de la Station centrale de Physiologie animale, 78 - Jouy-en-Josas qui a bien voulu effectuer ces dosages. 
Traitements témoins.

Implant bilatéral de benzoate d'œstradiol dans le cortex fronto-orbitaire ( 5 animaux) ou sous-cutané ( 2 animaux).

Implant bilatéral de cire dans l'hypothalamus (3 animaux).

\section{Expérience $I I$}

Au cours de cet essai, nous avons abordé en détail l'action des implants localisés à la zone où des réponses avaient été obtenues au cours de l'expérience préliminaire, ainsi que l'effet d'injections de progestérone.

Soixante-deux brebis ont été réparties en 4 groupes :

A : Trente-deux brebis ont reçu des implants bilatéraux de benzoate d'œestradiol au niveau de l'hypothalamus antérieur.

B: Seize brebis ont reçu des implants bilatéraux de cire, au niveau de l'hypothalamus antérieur.

C: Huit brebis ont reçu des implants bilatéraux de benzoate d'œestradiol dans le cortex cérébral.

D: Quatre brebis témoins n'ont pas été implantées.

Chaque groupe a été divisé en deux parties égales : les groupes $\mathrm{A}^{1}, \mathrm{~B}^{1}, \mathrm{C}^{1}$ et $\mathrm{D}^{1}$ ont reçu une injection intramusculaire de $20 \mathrm{mg}$ de progestérone par jour pendant 5 jours puis deux jours après la dernière injection, l'implant de benzoate d'œestradiol. Par la suite une série de 5 injections journalières de progestérone était répétée toutes les deux semaines. Les groupes $\mathrm{A}^{2}, \mathrm{~B}^{2}, \mathrm{C}^{2}$ et $\mathrm{D}^{2} \mathrm{n}^{\prime}$ ont reçu aucun traitement progestatif pendant cette phase de l'expérience qui a duré 18 semaines. A partir de ce moment, tandis que les traitements de progestérone étaient supprimés pour les groupes $\mathrm{A}^{1}, \mathrm{~B}^{1}, \mathrm{C}^{1}, \mathrm{D}^{1}$, les groupes $\mathrm{A}^{2}, \mathrm{~B}^{2}, \mathrm{C}^{2}$ et $\mathrm{D}^{2}$ ont reçu à 4 reprises des séries de 5 injections journalières intramusculaires de $20 \mathrm{mg}$ de progestérone espacées de 2 semaines.

\section{RÉSULTATS}

\section{Expérience $I$}

Les brebis ayant reçu un implant de benzoate d'œestradiol dans l'hypothalamus antérieur ont présenté dans 5 cas sur 7 une réponse d'œstrus (tabl. I) (fig. 2). Un seul cas par contre a été enregistré en ce qui concerne les implants plus postérieurs,

TABLEAU I

Réponses d'estrus chez des brebis ovariectomisées en fonction du lieu de l'implantation de benzoate d'ostradiol

\begin{tabular}{|c|c|c|c|c|}
\hline Groupe & Nature de l'implant & Lieu d'implantation & $\begin{array}{c}\text { Nombre } \\
\text { d'animaux }\end{array}$ & $\begin{array}{c}\text { Nombre } \\
\text { de répouses } \\
\text { d'œstrus }\end{array}$ \\
\hline I & Benzoate d'œstradiol & Sous-cutané (tête) & 2 & 0 \\
\hline II & Benzoate d'oestradiol & Cortex cérébral & 5 & 0 \\
\hline III & Cire & Hypothalamus médian & 3 & 0 \\
\hline \multirow[t]{3}{*}{ IV } & \multirow[t]{3}{*}{ Benzoate d'œstradiol } & Hypothalamus postérieur & 7 & 1 \\
\hline & & Hypothalamus médian & 9 & 0 \\
\hline & & Hypothalamus antérieur & 7 & 5 \\
\hline
\end{tabular}


et chez cet animal, une seule période de 3 jours d'œstrus a été observée aussitôt après l'implant, et aucune autre réponse n'a été enregistrée pendant les 6 mois suivants. A l'opposé, les brebis implantées dans l'hypothalamus antérieur ont présenté des périodes de comportement d'œstrus de durée variable et réparties sur l'ensemble des 6 mois d'observation suivant l'intervention.

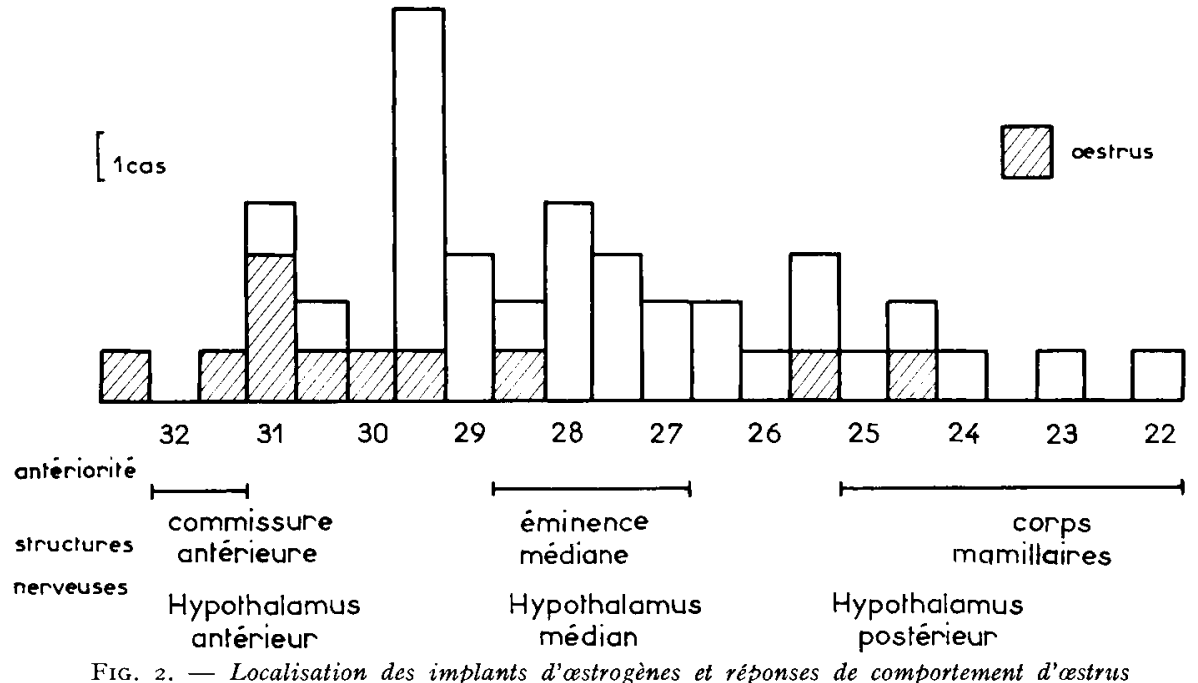

Chaque implant est reporté individuellement en fonction de la localisation établie par histologie. Sur

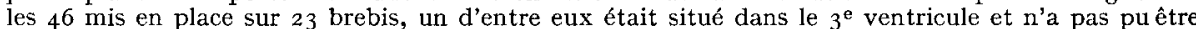
situé sur le schéma. Les implants correspondant à une femelle ayant présenté des réponses d'œstrus sont hachurés.

Chez les brebis ayant reçu un implant de benzoate d'œstradiol sous cutané ou dans le cortex cérébral, aucun comportement d'œstrus n'a été observé non plus que chez celles portant un implant hypothalamique de cire (groupes I, II et III).

\section{Expérience II}

\section{Apparition du comportement d'cestrus.}

Les résultats d'ensemble sont présentés dans le tableau 2. Sans progestérone un comportement d'œstrus est apparu chez 18 brebis sur les 32 ayant reçu un implant hypothalamique de benzoate d'œstradiol, alors qu'un seul cas d'œstrus était noté chez les femelles du groupe B (implant hypothalamique de cire) et deux chez celles du groupe $C$ (implant d'œstrogènes dans le cortex).

Après les injections de progestérone, la quasi totalité des brebis ayant reçu un implant d'œstrogènes vient en œstrus (30/32 dans le groupe $A ; 8 / 8$ dans le groupe C). Par contre pendant ces injections 8 femelles du groupe A continuent à présenter des cas d'œstrus tandis qu'un seul cas a été observé chez l'une des femelles portant un implant cortical.

Rythme d'apparition de l'cestrus.

L'apparition du comportement sexuel est nettement synchronisé avec la fin du traitement de progestérone (fig. 4), cependant, on peut remarquer que le début 
J.-P. SIGNORET

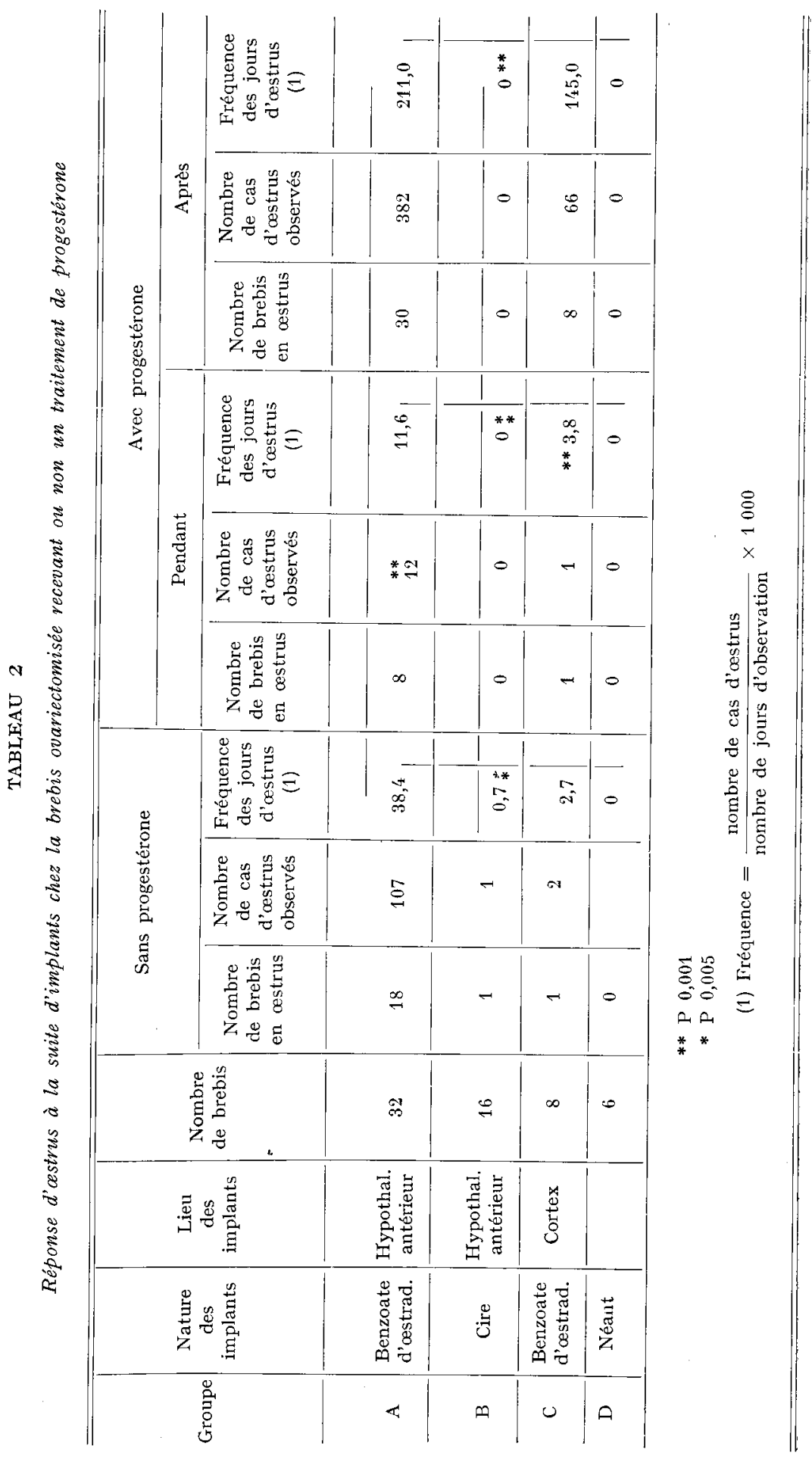


des ostrus est mieux groupé chez les femelles $\mathrm{C}$ que dans le groupe A (93,5 p. Ioo entre le $3^{\mathrm{e}}$ et le $6^{\mathrm{e}}$ jour contre $70,6 \mathrm{p}$. IO0, $\mathrm{P}<0,0 \mathrm{I}$ ), (tabl. 3 ).

Par contre, aucun rythme n'a été mis en évidence en ce qui concerne les intervalles entre les œestrus, que ce soit pendant les injections de progestérone ou en l'absence de celles-ci (fig. 3 et 4 ).

Enfin, la fréquence des réponses n'a pas varié sensiblement au cours des six mois de l'expérience.

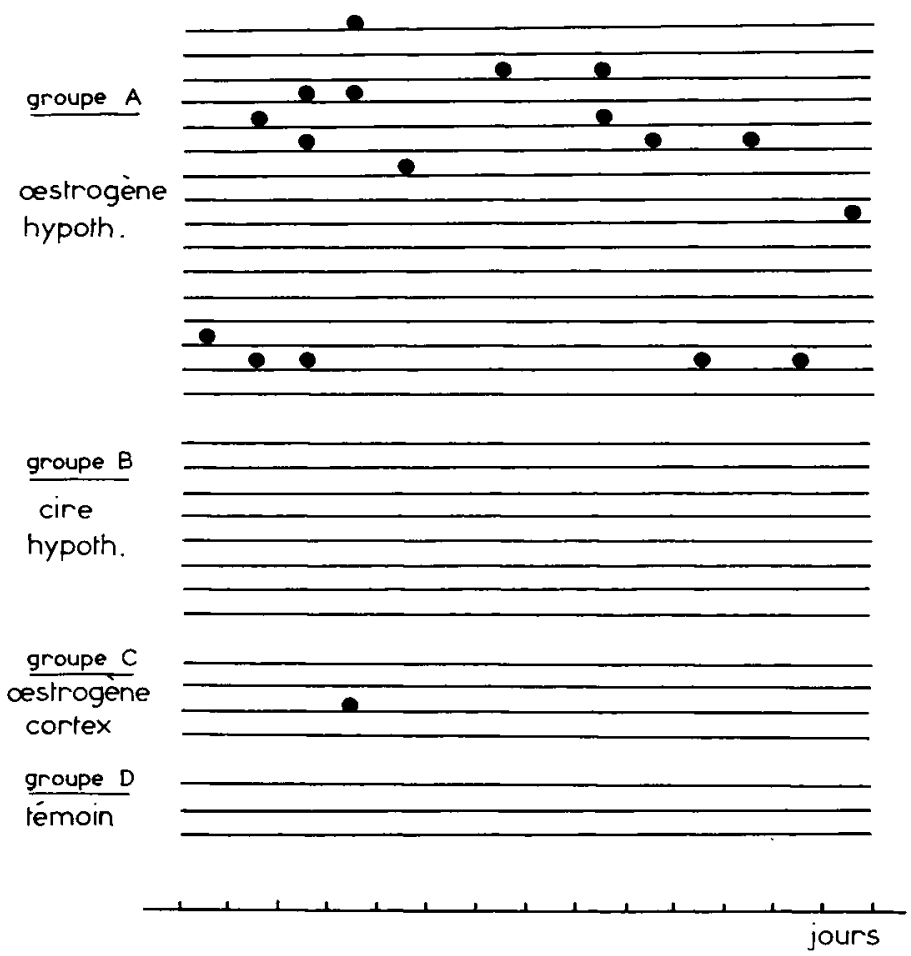

FIG. 3. - Exemple de répartition des véponses d'astrus en l'absence de traitement progestatif Chaque ligne représente une brebis

- Jour où a été observée une réponse d'œstrus

\section{TABLEAU 3}

Apparition du comportement sexuel après l'arrêt des injections de progestérone chez la brebis ovariectomisée ayant reçu un implant de benzoate d'ostradiol

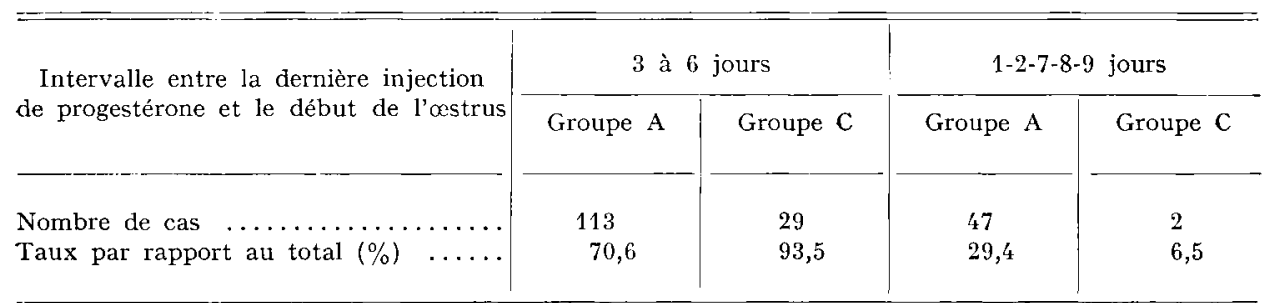

$$
\mathrm{X}^{2}=7,13 . \quad \mathrm{P} \text { inf. à } 0,01 \text {. }
$$




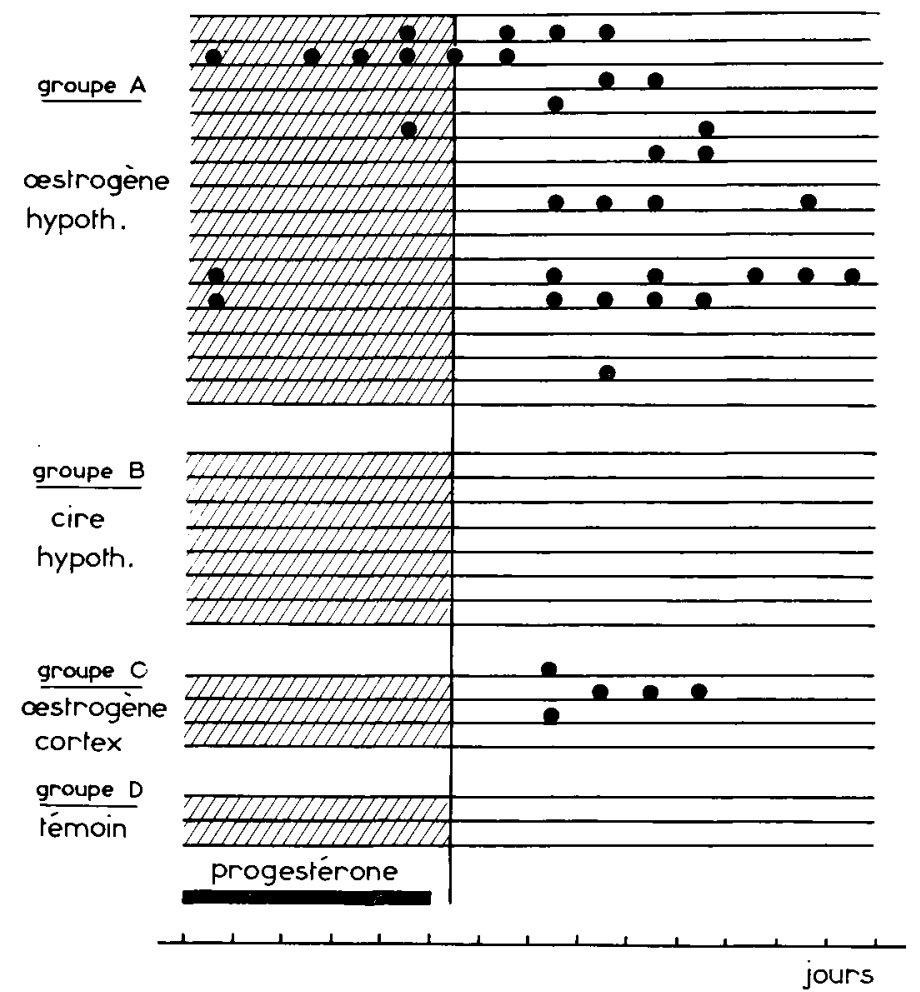

FrG. 4. - Exemple de répartition des réponses d'oestrus enregistrées pendant et après le traitement progestatif Chaque ligne représente une brebis.

- Jour où a été observée ure réponse d'œestrus

Estrogènes utilisés et action sur les organes crbles.

Le dosage chimique des œstrogènes restant sur les aiguilles après l'abattage a été effectué chez 7 animaux des lots $\mathrm{A}$ et $\mathrm{C} ; 73, \mathrm{I} 2 \mathrm{p}$. Ioo de la quantité totale a été utilisée pour un temps moyen d'implantation de six mois environ.

Si l'utilisation de l'hormone avait été régulière, la quantité libérée journellement dans l'organisme aurait été d'environ I3 microgrammes. Bien qu'il doive se produire avec le temps d'importantes variations dans la solubilisation du benzoate d'œstradiol implanté, ce chiffre donne un ordre de grandeur de la dose journalière reçue par l'animal.

La présence d'œstrogènes circulant a été mise en évidence par la variation du poids de l'utérus et les frottis vaginaux (tabl. 4) : où que soit situé l'implant d'œstrogènes, l'utérus accuse une augmentation de poids statistiquement significative tandis que les cellules recueillies dans le frottis vaginal sont toutes cornifiées. Au contraire, l'utérus et les frottis vaginaux des témoins portant un implant de cire sont analogues à ceux des femelles ovariectomisées non traitées.

Localisation des implants ayant provoqué des réponses d'cestrus.

La zone où les implants actifs ont été situés comprend l'ensemble de l'hypotha- 
TABLEAU 4

Action des implants de benzoate d'astradiol sur le poids utérin et les frottis vaginaux des brebis ovariectomisées

\begin{tabular}{|c|c|c|c|c|}
\hline Groupe & Nature de l'implant & Localisation & $\begin{array}{l}\text { Poids moyen } \\
\text { de l'utérus en } g\end{array}$ & $\begin{array}{c}\text { Frottis } \\
\text { vaginaux }\end{array}$ \\
\hline$A$ & $\begin{array}{l}\text { Benzoate } \\
\text { d'astradiol }\end{array}$ & Hypothalamus antérieus & $\begin{array}{r}25,23 \\
\pm \quad 0,91\end{array}$ & Cornifié \\
\hline $\mathrm{B}$ & Cire & Hypothalamus antérieur & $\begin{array}{r}11,62 \\
\pm \quad 0,91\end{array}$ & Repos \\
\hline $\mathrm{C}$ & $\begin{array}{l}\text { Benzoate } \\
\text { d'oestradiol }\end{array}$ & Cortex & $\begin{array}{r}20,70 \\
+\quad 2,29\end{array}$ & Cornifié \\
\hline $\mathrm{D}$ & Néant & Néant & $\begin{array}{r}10,29 \\
\pm \quad 1,87\end{array}$ & Repos \\
\hline
\end{tabular}

Total $\mathrm{F}=33,33$ (pour $1 \% \mathrm{~F}=4,13$ ).

$\mathrm{A}+\mathrm{CVSB}+\mathrm{DF}=47,25$ (pour $1 \%=4,98$ ).

lamus antérieur : aires préoptiques, aires septales, aires hypothalamiques antérieures (fig. 5).

Les implants actifs sont dispersés sur une zone s'étendant de $2,5 \mathrm{~mm}$ en avant de la commissure antérieure jusqu'à $I, 5 \mathrm{~mm}$ en arrière et latéralement entre $I$ et

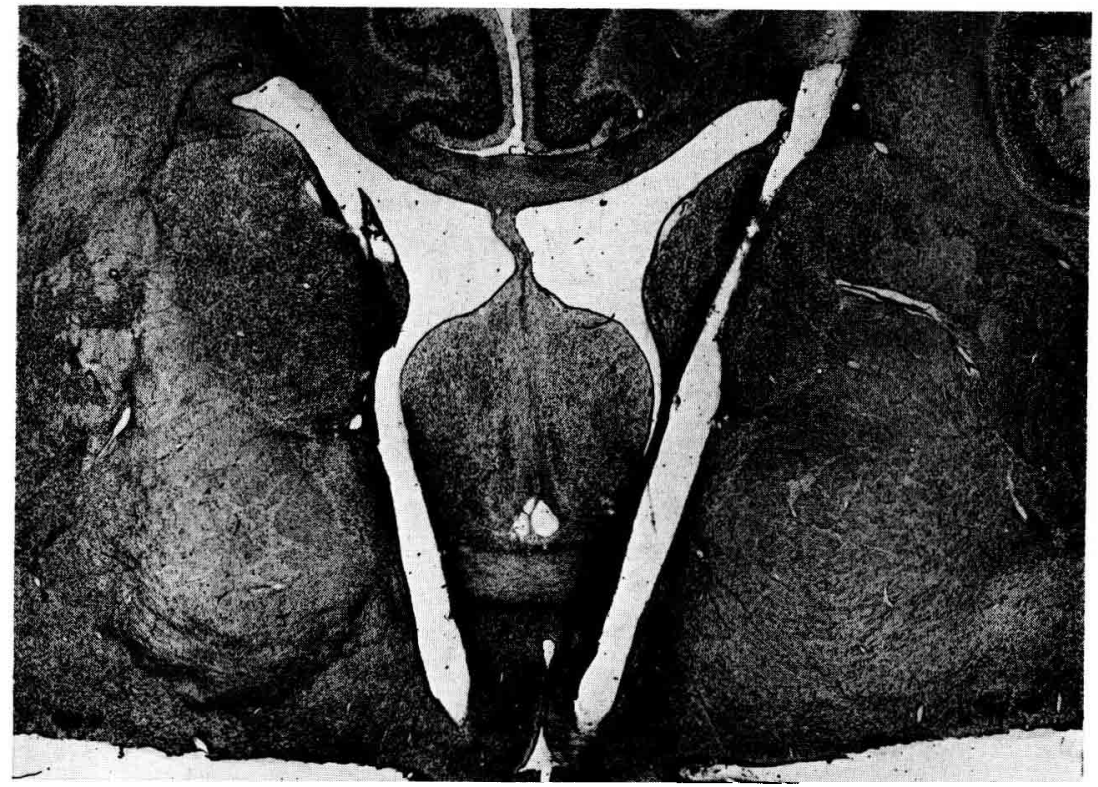

FIG. 5. - Localisation d'implants de benzoate d'astradiol induisant 'e comportement d'cestrus chez la brebis ovariectomisée 
$4 \mathrm{~mm}$ par rapport au plan de symétrie. Les taux de réponse sont analogues dans l'ensemble de cette zone, ce qui ne permet pas de relier l'action à une structure très précise. La localisation en antériorité de chaque implant d'après l'atlas établi au laboratoire est présentée dans les figures 6 a et $\mathrm{b}$.

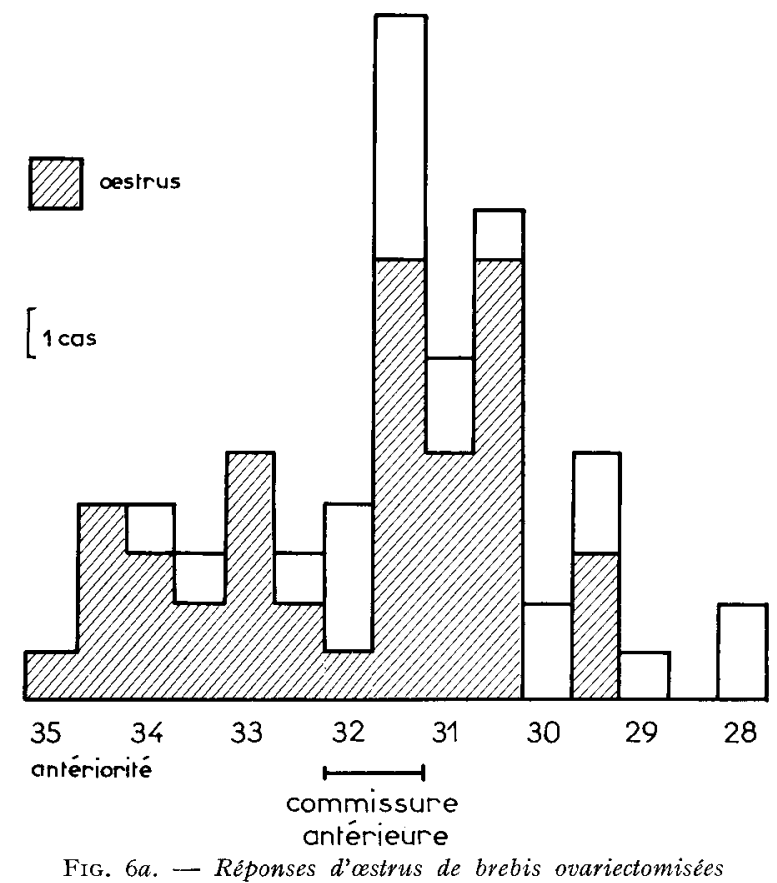

en fonction de l'emplacement des implants hypothalamiques de benzoate d'astradiol. Chaque implant est reporté individuellement en fonction de la localisation établie par histologie. Les cas correspondant à une réponse d'astrus sont hachurés.

\section{Comportement homosexuel.}

Un des résultats les plus frappants de l'implantation d'œestrogènes dans les structures cérébrales a été l'apparition chez la femelle de tous les éléments moteurs de comportement sexuel mâle, tels qu'ils sont décrits chez les Ovins par Banks (rg64) ou chez les Ongulés sauvages d'espèces voisines (WAL'THER, I958 ; BACKHAUS, I958) : position de recherche tête basse (fig. 7), flairage de la région anogénitale, réaction ritualisée de " moue " ("flehmen " des auteurs allemands) (fig. 9), approche arrière, la tête placée latéralement sur le flanc de la femelle, ce mouvement étant en général accompagné d'un coup de patte et d'une émission sonore (fig. 8), chevauchement (fig. Io), coup de tête en arrière analogue à la réaction qui chez le mâle accompagne l'éjaculation.

Le tableau 5 montre que la fréquence de ces manifestations est plus élevée dans le groupe ayant reçu l'implant de benzoate d'œestradiol dans l'hypothalamus par rapport à celui où il était localisé dans le cortex; bien que rarement, on remarque quelques cas de tels comportements chez les témoins non traités ou ayant reçu un implant de cire. Toutefois, ces réactions n'ont pas été enregistrées chez des femelles normales qu'elles soient en œestrus ou non (tab1. 6). 


\section{TABLEAU 5}

Fréquence des comportements pseudo-mâles après implantation de benzoate d'aestradiol dans l'hypothalamus antérieur et le cortex de brebis ovariectomisées

\begin{tabular}{|c|c|c|c|c|c|c|c|}
\hline \multirow[b]{2}{*}{ Groupe } & \multirow[b]{2}{*}{$\begin{array}{c}\text { Nombre } \\
\text { total }\end{array}$} & \multicolumn{2}{|c|}{ Sans progestérone } & \multicolumn{2}{|c|}{$\begin{array}{l}\text { Pendant le traitement } \\
\text { de progestérone }\end{array}$} & \multicolumn{2}{|c|}{$\begin{array}{l}\text { Après le traitement } \\
\text { de progestérone }\end{array}$} \\
\hline & & $\begin{array}{l}\text { Nombre } \\
\text { de femelles } \\
\text { préseutaut uı } \\
\text { comportement } \\
\text { pseudo-mâle }\end{array}$ & $\begin{array}{l}\text { Fré- } \\
\text { quence } \\
(1)\end{array}$ & $\begin{array}{l}\text { Nombre } \\
\text { de femelles } \\
\text { présentant un } \\
\text { comportement } \\
\text { pseudo-mâle }\end{array}$ & $\begin{array}{l}\text { Fré- } \\
\text { quence } \\
(\mathbf{1})\end{array}$ & $\begin{array}{l}\text { Nombre } \\
\text { de femelles } \\
\text { présentant un } \\
\text { comportement } \\
\text { pseudo-mâle }\end{array}$ & $\begin{array}{l}\text { Fré- } \\
\text { quence } \\
\text { (1) }\end{array}$ \\
\hline A & 32 & 25 & 747 & 11 & 309 & 15 & 430 \\
\hline B & 16 & 2 & 34 & 1 & 19 & 2 & 21 \\
\hline $\mathrm{C}$ & 8 & 3 & 335 & 2 & 115 & 4 & 329 \\
\hline $\mathrm{D}$ & 6 & 4 & 13 & 0 & 0 & 2 & 66 \\
\hline
\end{tabular}

(Pour la définition des groupes, se reporter au tableau 2).

(1) Fréquence $=\frac{\mathrm{Nb} \text { de jours avec manif. pseudo-mâle }}{\mathrm{Nb} \text { de jours d'observations }} \times 1000$

TABLEAU 6

Réactions pseudo-mâles dans un groupe de brebis Ile-de-France non traitées

\begin{tabular}{c|c|c|c|c}
$\begin{array}{c}\text { Nombre de jours } \\
\text { d'observations } \\
(2 \mathrm{~h} / \mathrm{j})\end{array}$ & $\begin{array}{c}\text { Nombre } \\
\text { de femelles }\end{array}$ & $\begin{array}{c}\text { Nombre de femelles } \\
\text { venues en oestrus }\end{array}$ & $\begin{array}{c}\text { Nombre de jours } \\
\text { de femelle } \\
\text { en oestrus } \\
32\end{array}$ & $\begin{array}{c}\text { Nombre de cas } \\
\text { de comportement } \\
\text { pseudo-mâle } \\
\text { observés }\end{array}$ \\
\hline
\end{tabular}

\section{DISCUSSION ET CONCLUSION}

L'utilisation d'un cestrogène présentant une certaine solubilité comme le benzoate d'œstradiol peut laisser craindre qu'une action systémique de l'hormone ne risque de fausser les conclusions relatives à l'effet local de l'implant. La dose journalière libérée pendant la totalité de l'expérience est très élevée puisqu'elle est du même ordre de grandeur que celle qui en une seule injection intramusculaire provoque la réponse d'œestrus chez $50 \mathrm{p}$. Ioo des brebis prétraitées à la progestérone (RobINSON et REARDON, I96r ; ROBINSON et BRANDER, I962).

L'action sur les organes cibles — utérus et vagin — montre la présence d'œs- 
ABRÉVATIONS

(d'après Nomina anatomica, Paris I955)

AHL

AHA

APO

A. Sept. I.

A. Sept. M.

$\mathrm{CA}$

$\mathrm{CC}$

$\mathrm{Cd}$

$\mathrm{ChO}$

C. Int.

$\mathrm{Cl}$

FM'T

$\mathrm{Fx}$

NHA

NHDM

NHVM

N. inf.

NPOMd

NPV

NSO

Put

ROI

$\mathrm{SCh}$

TO

TO1
Area hypothalamica lateralis

Area hypothalamica anterior

Area preoptica

Area septalis lateralis

Area septalis medialis

Commissura anterior

Corpus callosum

Nucleus caudatus

Chiasma opticum

Capsula interna

Claustrum

Fasciculus mamillo thalamicus

Fornix

Nucleus hypothalamicus anterior

Nucleus hypothalamicus dorsalis medialis

Nucleus hypothalamicus ventralis medialis

Nucleus infundibularis

Nucleus preopticus medialis

Nucleus paraventricularis hypothalami

Nucleus supraopticus

Putamen

Radiatio olfactoria interna

Nucleus suprachiasmaticus

Tractus opticus

Tractus olfactorius 

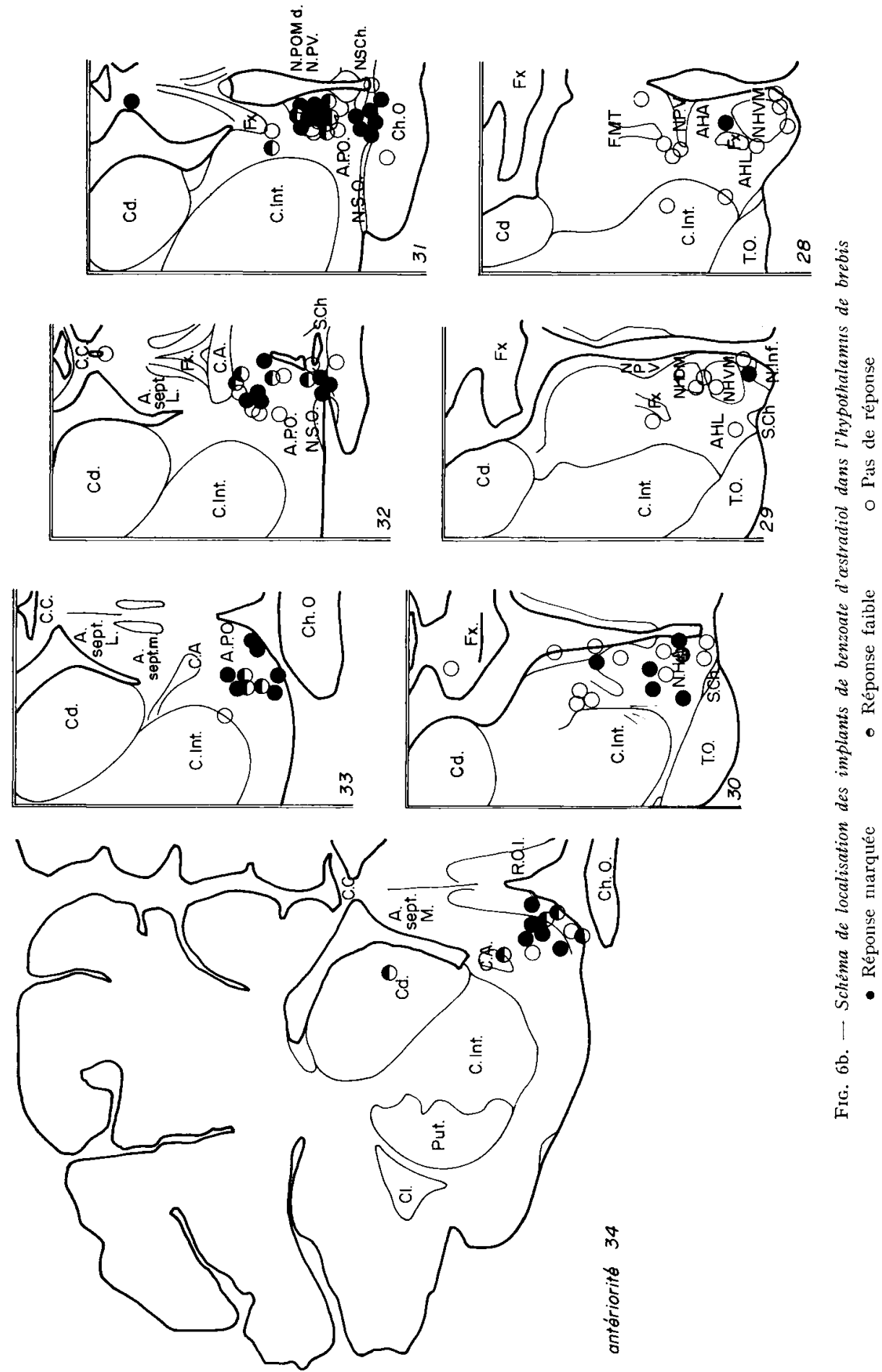

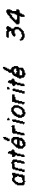



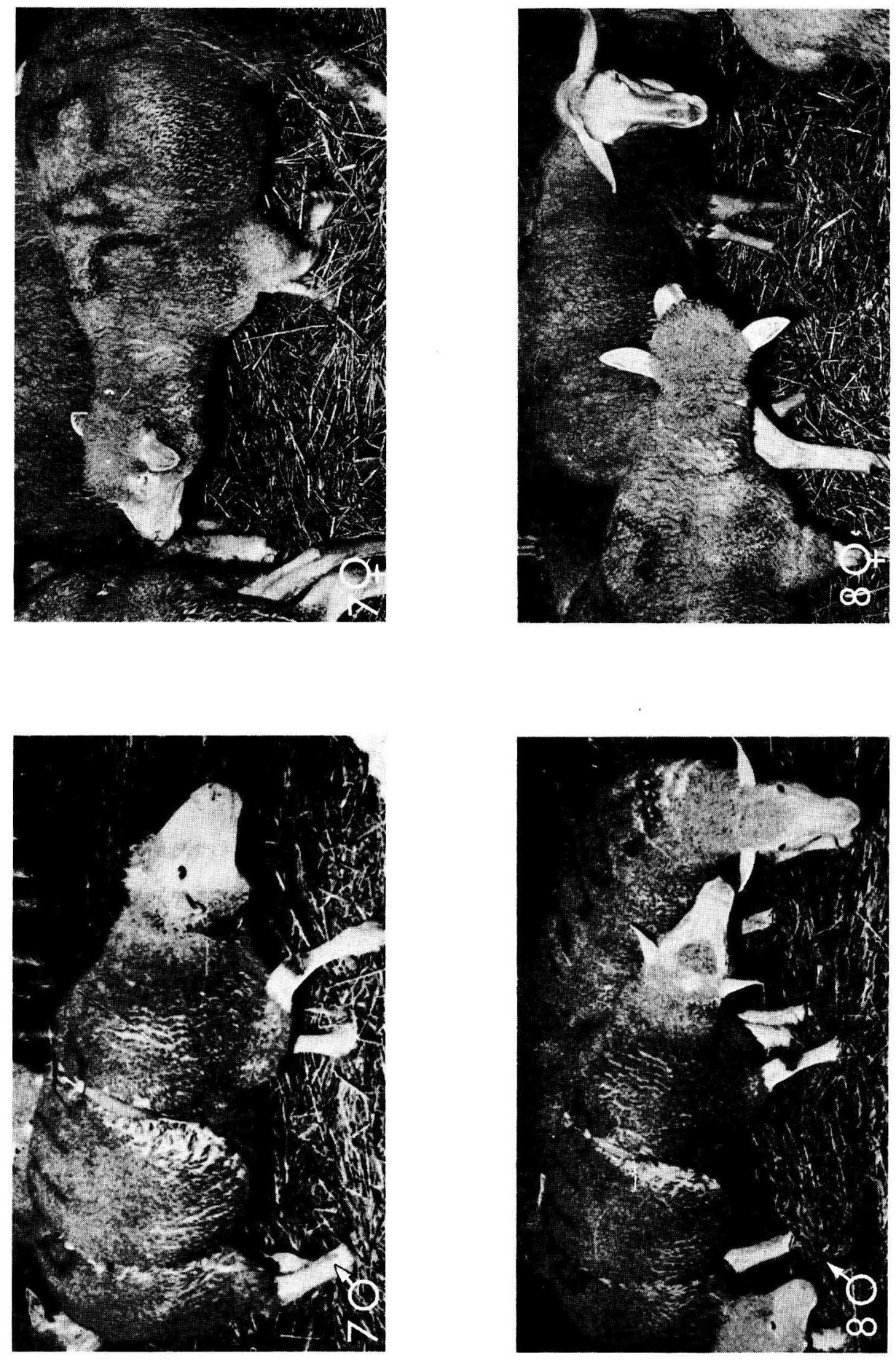

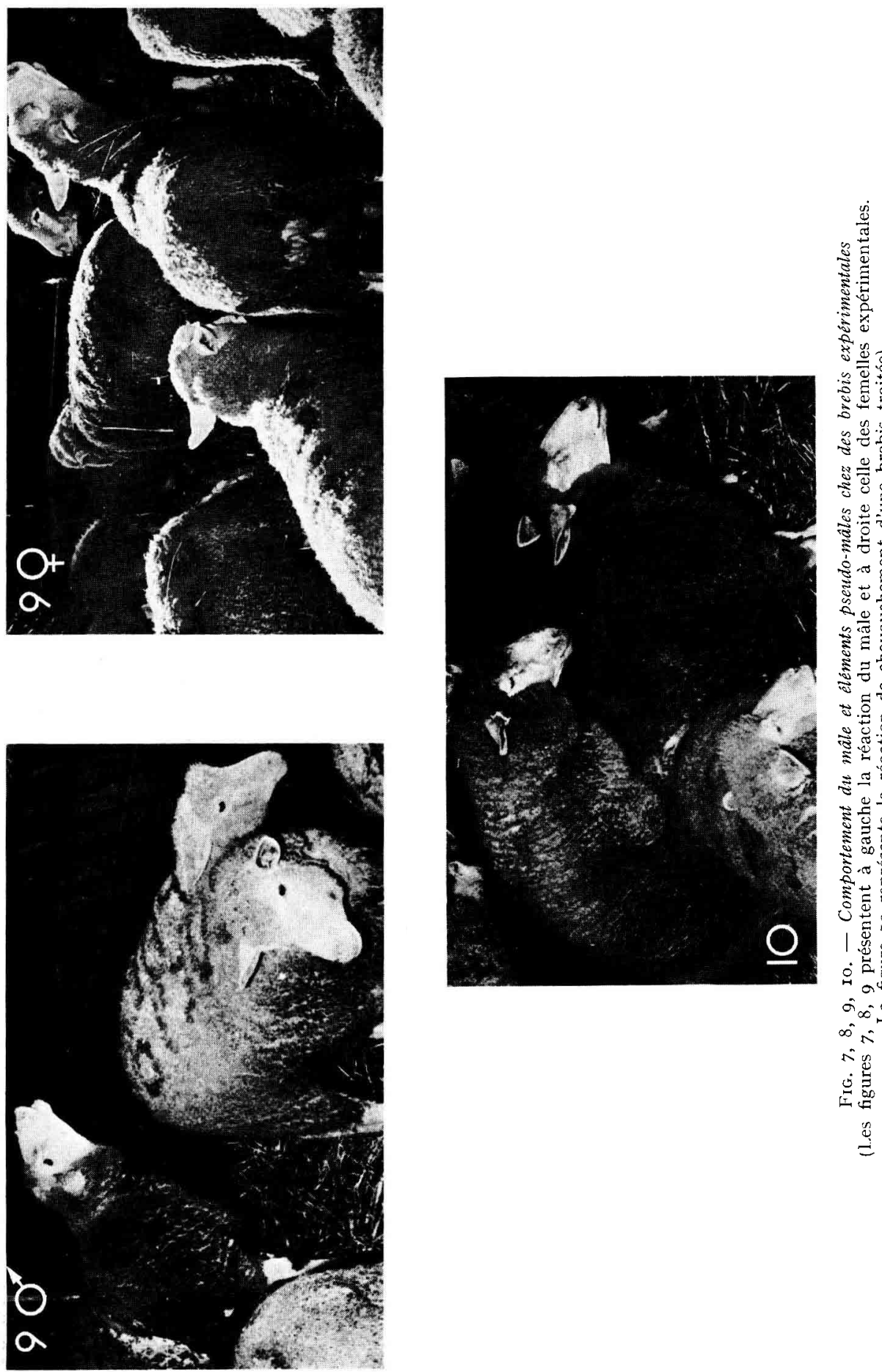

害过

事要要

$5+$

$2 \frac{9}{3}$

s

ड़

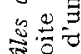

焉步

$\stackrel{5}{8}$

究

Q 0

요

究焉

จ

จ

今

政

폰

요요

농

용

के

농

递。

总苛

कि

$\infty \therefore$

nin

总焉

$\stackrel{0}{=}$ 
trogènes circulant en dose physiologiquement active chez les animaux ayant reçu un implant aussi bien dans l'hypothalamus que dans le cortex.

Il est donc normal que dans ces conditions, des réponses de comportement sexuel apparaissent après l'interruption d'un traitement progestatif, aussi bien chez les brebis du groupe A (implant hypothalamique) que chez celles du groupe C (implant cortical). En effet, l'effet facilitant des injections de progestérone permet aux œstrogènes circulant de déclencher le comportement d'œestrus.

Par contre, si la libération constante de doses faibles d'œestrogènes peut à la longue provoquer l'apparition du comportement sexuel chez le Cobaye (DAvidson et al., I968), l'obtention de telles réponses après traitement intramusculaire d'œestrogènes seuls requiert rapidement chez la Brebis des doses très importantes — jusqu'à plusieurs milligrammes - (ROBINson, I954). De même, des injections journalières de 50 microgrammes de benzoate d'œstradiol ne produisent de réponses d'œstrus que les quelques premiers jours du traitement (SigNorEiT, données non publiées). Cependant, en l'absence de progestérone, la fréquence des réponses de comportement sexuel est très supérieure $(\mathrm{P}<\mathrm{o}, \mathrm{OoI})$ lorsque l'implant de benzoate d'œstradiol est situé dans 1'hypothalamus antérieur préoptique et supraoptique. De même, pendant les injections de progestérone, qui empêchent l'apparition du comportement d'ostrus chez la femelle castrée (MOORE et ROBINSON, I957) seuls les implants hypothalamiques provoquent des réponses. On peut donc émettre 1'hypothèse que le benzoate d'œstradiol diffusant à partir de l'implant dans les tissus et par les vaisseaux sanguins atteindrait une concentration locale très supérieure à celle qui suit une injection intramusculaire. Lorsque 1'implant est localisé à une zone où la densité des cellules sensibles aux ostrogènes est la plus forte, on pourrait expliquer les réponses d'œestrus indépendamment de l'effet facilitant d'une préparation progestative ou de l'action inhibitrice d'un traitement simultané de progestérone. Ainsi les zones hypothalamiques antérieures correspondraient chez la Brebis comme chez le Rat (LISK, I962, I965) à la région où agissent les œstrogènes pour déclencher le comportement sexuel.

La présence d'œstrogènes, mais à une dose plus faible parce que l'implant est plus éloigné des structures sensibles expliquerait la réponse moins marquée observée chez les témoins ayant reçu des implants dans le cortex cérébral.

Enfin, en ce qui concerne les témoins ayant reçu un implant de cire à trépanation, l'absence de comportement et d'œstrus montre que la lésion produite n'est pas en cause.

L'absence de relations précises avec une structure anatomique bien caractérisée, concorde avec les résultats de GLAScock et MichaEL (I962) qui, chez le Chat, ont montré l'existence de cellules fixant les œestrogènes marqués dispersés dans les aires hypothalamiques et non regroupées en un noyau identifiable.

On pourrait émettre une hypothèse analogue en ce qui concerne l'apparition du comportement sexuel pseudo-mâle : chez les femelles des mammifères, on note en général la présence des différents éléments moteurs du comportement sexuel mâle ainsi que l'augmentation de ces manifestations dans le comportement sexuel normal (BEACH, I968). Par contre, ces manifestations sont exceptionnelles chez la Brebis, même dans le cas d'injections journalières de benzoate d'œstradiol poursuivies pendant un mois (Signoret, non publié). Or, on peut penser que, dans le cas de notre expérience, la libération lente et continue d'œstrogène dans les struc- 
tures cérébrales amène localement une concentration d'hormone très supérieure à celle qui atteint ces structures lorsque des doses physiologiques sont apportées par la voie systémique. On pourrait ainsi atteindre un seuil où apparaîtrait le comportement pseudo-mâle, tandis que chez d'autres espèces de mammifères les doses physiologiques seraient suffisantes pour mettre en œuvre ce mécanisme.

Le taux plus élevé de réponses à la suite d'implants hypothalamiques antérieurs permettrait d'émettre l'hypothèse que les mêmes aires cérébrales seraient impliquées dans la mise en ouvre des comportements mâle et femelle. Par contre, bien que 1a fréquence en soit faible, l'apparition de réactions pseudo-mâles chez les témoins ayant reçu un implant de cire ou non traités, est difficile à interpréter. Il pourrait s'agir d'un comportement " de jeu " provoqué par une activité anormale dans le groupe de brebis. En effet, la seule occasion qui nous a été donnée d'observer un tel comportement en dehors de cette expérience se situait lors de l'activité de jeu chez des brebis d'un an en période d'anœestrus à la sortie d'un parc : alors que les animaux effectuaient des courses rapides entrecoupées de bonds, plusieurs chevauchements furent notés ainsi que des luttes tête-à-tête. Le comportement anormal des femelles traitées aux œestrogènes pourrait ainsi avoir induit la réaction des témoins. En effet, chez les Bovins, Sambraus (1969) signale une augmentation de la fréquence des chevauchements des femelles en anœstrus lorsque dans le groupe, une vache est en chaleur et présente de ce fait une activité de monte intense.

En conclusion : à défaut d'un "centre du comportement sexuel ", qui soit bien défini anatomiquement, nous avons pu mettre en évidence dans l'hypothalamus antérieur une zone privilégiée pour l'action des œstrogènes. En outre l'apparition d'un comportement pseudo-mâle à la suite d'implant dans cette zone confirme, chez la Brebis, où ces réactions sont exceptionnelles spontanément, la présence générale des deux schémas de comportement sexuel mâle et femelle, et leur possibilité de mise en ouvre simultanée par une même hormone.

Reçu pour publication en septembre 1970.

\author{
SUMMARY \\ EFFECT OF HYPOTHALAMIC IMPLANTS OF GESTRADIOL BENZOATE \\ ON CESTROUS BEHAVIOUR IN THE OVARIECTOMIZED EWE
}

Oestradiol benzoate fused to stainless steel tubes was implanted in the hypothalamus of ovariectomized ewes intramuscularly injected or not with progesterone.

Though uterus weight gain and vaginal keratinization give evidence for a systemic action of oestradiol benzoate, oestrous responses from either non progesterone-injected ewes or ewes with progesterone treatment in progress were induced only by implants located in the anterior preoptic area. However all oestrogen implanted animals exhibit sexual response two days after cessation of the progesterone treatment, accounting for the facilitating effect of progesterone.

Male behavioral reactions were observed in most implanted ewes, whereas male or female sexual behaviour hardly occured in the controls (cortical implants or hypothalamic sham implants)

For want of a definite centre, at least a responsive area for oestrogen action was located in the hypothalamus. Inducing male behavioral reactions, which are most uncommon in the ewe, further supports the evidence of both male and female behavioral patterns in the brain and their potential induction by the same hormone. 


\section{RÉFÉRENCES BIBLIOGRAPHIQUES}

Backнaus D., 1958. Beitrag zur Ethologie der Paarung einiger Antilopen. Zuchthyg., 2, 28 r-293.

BANks E. M., I964. Some aspects of sexual behaviour in domestic sheep (Ovis aries). Behaviour, 23, $249-279$.

$\mathrm{BEACH}$ F. A., I942. Importance of progesterone to induction of sexual receptivity in spayed female rats. Proc. Soc. Exp. Biol. Med., 51, 369-3I7.

BEAcr F. A., I968. Factors involved in the control of mounting behavior by female mammals. In : Perspectives in reproduction and sexual behavior. M. Diamond ed. p. 83-131, Indiana Univ. Press, Bloomington-London.

Boling J. L., Blandau R. J., 1939. The ostrogen-progesterone induction of mating responses in the spayed female rat. Endocrinology, 25, 359-364.

Davidson J. M., Smith E. R., Rodgers C. H., Block G. J., I968. Relative thresholds of behavioral and somatic responses to oestrogens. Physiol. Behav., 3, 227-229.

Glascock R. F., Michael R. P., I962. The localization of oestrogen in a neurological system in the brain of the female cat. (Proc.) J. Physiol., 163, 38 P-39 P.

Harris G. W., Michael R. P., ig64. The activation of sexual behaviour by hypothalamic implants of œstrogen. J. Physiol., 171, 275-30I.

Harris G. W., Michael R. P., r958. Hypothalamic mechanism and the control of sexual behavior in the female cat. J. Physiol., 142, $26 \mathrm{P}$ (proc.).

ITtRICH G., 1958. Eine neue Methode zur chemischen Bestimmung der östrogen Hormone in Ham. Hoppe Seylers Z. Physiol. Chemic., 312, I-I4.

ITTRICH G., I960. Untersuchungen über die Extraktion des roten Kober Farbstoffe durch organische Lösungsmittel zur Östrogen Bestimmung im Harn. Acta Endocr., 35, 34-48.

Lisk R. D., I962. Diencephalic placement of estradiol and sexual receptivity in the female rat. Amer. J. Physiol., 203, 493-496.

Lrsk R. D., I965. Reproductive capacity and behavioural ostrus in the rat bearing hypothalamic implants of sex steroids. Acta Endocr., 48, 209-219.

Lisk R. D., Wein A. J., I962. Neurological site of action of estradiol in eliciting estrous behavior in the spayed rat. (Abstr.) Amer. Zool., 2, 426.

Michael R. P., I96I. An investigation of the sensitivity of circumscribed neurological areas to hormone stimulation by means of the application of ostrogens directly to the brain of the cat. 4th Intern. Neurochemical Symposium, Pergamon Press. London N. Y., p. 465-48o.

Michael R. P., I962. Estrogen-sensitive neurons and sexual behavior in female cats. Science, 136, $322-323$.

Mrchael R. P., ScotT P. P., I957. Quantitative studies on mating behaviour of spayed female cats stimulated by treatment with oestrogens. J. Physiol, 138, 46-47.

Michael R. P., ScotT P. P., I964. The activation of sexual behaviour in cats by the subcutaneous administration of estrogen. J. Physiol., 171, 254-274.

Moore N. W., Robinson T. J., I957. The behavioral and vaginal response of the spayed ewe to estrogens injected at various times relative to the injection of progesterone. $J$. Endocr., 15, $360-365$.

PALKA Y. S., SAWYer C. H., I964. Induction of cestrous behavior in the ovariectomized rabbit by cestrogen implants in the hypothalamus. (Abstr.) Amer. Zool., 4, 289.

Palka Y.S., Sawyer C. H., I966. The effects of hypothalamic implants of ovarian steroids on cestrous behaviour in rabbits. $J$. Physiol., 185, $25 \times-269$.

RoBinson T. J., 1954. Relationship of cestrogen and progesterone in ostrous behaviour of the ewe. Nature, 173, 878 .

Robinson T. J., REARdon T. F., I96I. The activity of a number of oestrogens as tested in the spayed ewe. J. Endocr., 23, 97-I07.

Robinson T. J., Brander W. M., I962. Comparative activities of a number of cestrogens when administered intravenously and intramuscularly in the sheep. J. Reprod. Fert., 3, 74-88.

SAWYER C. H., I963. Induction of estrus in the ovariectomized cat by local hypothalamic treatment with estrogens. (Abstr.) Anat. Rec., 145, 280.

Walther F., 1958. Zum Kampf und Paarungs Verhaltens einiger Antilopen. Zeitsch. Tierpsychol, 15, $340-380$. 